\title{
A importância dos processos gerenciais nos resultados de produção mais limpa: Um estudo na indústria do plástico
}

\author{
The importance of management processes in cleaner production results: A study in \\ the plastic industry
}

\author{
Dalton Oswaldo Buccelli'; Pedro Luiz de Oliveira Costa Neto' \\ ' Programa de Pós-Graduação em Engenharia de Produção, Universidade Paulista - UNIP, SP. Brasil
}

\begin{abstract}
Resumo
O artigo relata um estudo sobre a introdução do programa de produção mais limpa na indústria do plástico. Seu objetivo principal foi o desenvolvimento de um modelo de avaliação da qualidade da gestão ambiental para comprovar a proposição de que os resultados ambientais permanentes de produção mais limpa tem uma relação positiva com a existência de processos gerenciais estruturados e padronizados nas empresas. Uma revisão da literatura permitiu o desenvolvimento do modelo de avaliação da estruturação e padronização dos processos gerenciais, utilizando-se como referência a literatura existente sobre modelos de negócios e gestão por processos, assim como os modelos de excelência em gestão adotados pelos prêmios nacionais de qualidade de diferentes países e continentes. A proposição da existência de uma relação positiva entre os processos gerenciais estruturados e o desempenho ambiental das empresas foi confirmada por meio de pesquisa quantitativa realizada com 32 empresas associadas ao sindicato da indústria do plástico de São Paulo - SINDIPLAST. A obtenção de resultados ambientais significativos em decorrência da incorporação de princípios e conceitos de produção mais limpa nos processos gerenciais abre um amplo caminho para novos estudos em outras localidades e em outros setores da indústria.
\end{abstract}

Palavras-chave: Produção mais limpa, Indicadores ambientais, Processos gerenciais, Indústria do plástico.

\begin{abstract}
The paper reports a study on the implementation of cleaner production in the plastic industry. The study aimed to develop a model for assessing the quality of environmental management to prove the proposition that permanent cleaner production outcome has a positive relationship with the existence of structured and standardized management processes in the company. A literature review allowed the development of a model to evaluate the degree of structuring and standardization of management processes, using as reference the existing literature on business models and process management, as well as excellence models adopted by national quality awards from different countries and continents. The proposition of a positive relationship between the structured management processes and environmental performance of the companies was confirmed by quantitative survey with 32 companies' members of the São Paulo plastic industry association - SINDIPLAST. These findings on significant environmental results from the incorporation of principles and concepts of cleaner production in management processes will allow to do further studies in other locations and other industry sectors.
\end{abstract}

Keywords: Cleaner production, Environmental indicators, Management processes, Plastic industry. 


\section{INTRODUÇÃO}

É fato que o crescimento econômico sempre ignorou o meio ambiente, mas consumidores, fornecedores, governos e mercado em geral estão cada vez mais exigentes com a comunidade empresarial no que diz respeito à responsabilidade ambiental. Como um impacto da globalização e do ambiente competitivo a tomada de medidas imediatas e definitivas relacionadas às questões ambientais surge como uma nova demanda para os gestores ligados à indústria. Faz-se necessária a implementação de estratégias que melhorem continuamente a qualidade, a capacidade e a eficiência dos produtos e dos processos produtivos reduzindo desperdícios de recursos e o consumo de materiais.

De acordo com Ehrlich e Ehrlich (2010), a humanidade precisa encontrar formas de mudar suas atitudes em relação ao que é mais essencial ao perfeito funcionamento da natureza. Todos deveriam ser capazes de reconhecer as causas básicas da degradação ambiental e de compreender que o crescimento infinito da economia é fisicamente impossível. Os autores observam, de maneira simples, que o impacto negativo do ser humano nos ecossistemas naturais é função de três fatores básicos: o aumento no tamanho da população, o crescimento desregrado do consumo per capita e a escassez cada vez maior de recursos naturais necessários para suprir o consumo humano. Dos fatores apontados, a mudança nos hábitos de consumo seria a maneira viável de minimizar a degradação dos ecossistemas naturais. No entanto, o consumo sustentável continua a ser um tema relativamente desconhecido para a grande maioria da população, mesmo nas grandes metrópoles onde a informação e o conhecimento sobre as questões ambientais são abundantes.

Hart (1997) argumenta que para se alcançar uma economia sustentável no futuro é imprescindível a redução do impacto negativo causado pela indústria ao meio ambiente. A solução passa por uma mudança nas tecnologias usadas para produzir bens e serviços, que tragam maior eficiência aos produtos e aos processos produtivos, reduzindo custos e mitigando impactos ambientais. No entanto, nos países em desenvolvimento, foram poucas as empresas que incorporaram a sustentabilidade em seu pensamento estratégico. A estratégia ambiental ainda consiste em patrocinar pequenos projetos destinados a controlar a poluição. A transição para sociedades mais sustentáveis está ligada ao uso mais eficiente e consciente de matérias-primas, de fontes renováveis e não renováveis de energia e ao desenvolvimento de novas tecnologias que permitam a redução de emissões e impactos nos ecossistemas (Bonilla et al, 2010).

A prevenção ambiental integrada aos processos, produtos e serviços para aumentar a eficiência e reduzir os riscos para os seres humanos e o meio ambiente é um dos propósitos da aplicação dos conceitos de produção mais limpa. Ela traz melhorias em diversos aspectos dos processos principais da cadeia de valor das empresas e em seus processos de apoio, como: redução do consumo de energia elétrica, redução do consumo de água, diminuição dos índices de refugo (material para reprocesso e reciclagem), diminuição dos índices de resíduos e melhoria da ecoeficiência.

No Brasil, alguns pioneiros na indústria, envolvendo pequenas e médias empresas responsáveis por uma parte importante do crescimento econômico e da criação de emprego no país, começaram a se reunir com órgãos do governo em 1998, surgindo uma parceria entre a agência reguladora, Companhia Ambiental do Estado de São Paulo (CETESB) e representantes do setor industrial, como Federação das Indústrias do Estado de São Paulo (FIESP) e seus sindicatos filiados. O projeto começou com atividades de atualização de conceitos e programas para sensibilizar as pessoas para o potencial de redução de custos e de aumento de produtividade por meio da integração de práticas ambientalmente sustentáveis na rotina de seus processos de negócio.

Até os dias de hoje foram escritos mais de 16 guias técnicos setoriais, contendo os fluxogramas dos processos produtivos de cada indústria e as oportunidades para implantação de soluções de produção mais limpa. Além disso, a CETESB incentivou a publicação de casos de sucesso em seu portal na internet, em que as empresas descrevem as medidas implementadas que obtiveram resultados favoráveis para sustentabilidade econômica, social e ambiental. Somente 70 casos de sucesso foram publicados, abrangendo ações implantadas em alguns setores da indústria paulista, principalmente metal-mecânico (46\%), químico/farmacêutico (20\%), galvanoplastia (14\%) e têxtil (9\%). A concepção do programa envolvendo sensibilização, capacitação, participação de representantes dos setores e demonstração de casos de sucesso foi incapaz de atingir um aumento no número de publicações de histórias de sucesso de produção mais limpa.

A estratégia utilizada para motivar outros líderes industriais na esperança de gerar reações em 
cadeia baseou-se na implantação de projetos técnicos ligados aos processos operacionais. Portanto, esta experiência sugere que projetos de benchmarking têm resultados limitados na promoção da produção mais limpa em empresas de médio e pequeno porte, nos diversos tipos de setores industriais envolvidos. Ashton et al (2002) consideram a participação em avaliações e premiações uma abordagem inovadora para a melhoria da conscientização sobre produção mais limpa dentro da empresa, num determinado setor industrial ou no nível nacional. De acordo com Vendrametto et al (2010), é melhor usar uma combinação de estratégias, além do treinamento e da apresentação de casos de sucesso no setor industrial. Iniciativas de implantação de produção mais limpa foram mais eficazes em empresas que já haviam experimentado outros métodos de melhoria de processos produtivos, como o Total Quality Control e o Lean Manufacturing. As organizações de classe mundial buscam estruturar primeiramente seus processos gerenciais como uma forma de tornar as iniciativas sólidas e permanentes.

Levando-se em conta estas afirmações, pode-se inferir que a presença de conceitos e fundamentos da produção mais limpa nos processos de gestão da empresa é essencial para a efetiva execução de melhorias em produtos e processos de negócio. A incorporação dos fundamentos nos processos gerenciais contribui para a identificação e implantação das oportunidades de produção mais limpa na cadeia de valor, permitindo obter resultados favoráveis e em patamares cada vez mais elevados. Consequentemente, este trabalho trata do relacionamento existente entre bons resultados de indicadores ambientais e a introdução de conceitos e princípios da produção mais limpa nos processos gerenciais estruturados e padronizados das empresas do setor industrial de transformação de materiais plásticos do Estado de São Paulo.

\section{I.I Processos do Negócio}

Os autores Rummler e Brache (1995) subdividem as atividades existentes em qualquer empresa em três tipos de processos do negócio: primários (processos que pertencem à cadeia de valor e geram os produtos e serviços que atendem o cliente), auxiliares (que suportam os processos primários do negócio) e gerenciais (processos que regem o funcionamento da empresa).

Os processos primários se preocupam em vender, produzir, entregar, dar assistência técnica, aperfeiçoar e, quando necessário, descontinuar o produto. Os processos auxiliares se preocupam em comprar materiais, controlar os recursos para a execução do produto, recrutar e selecionar pessoal, fazer a contabilidade atendendo às exigências governamentais e manter em perfeito funcionamento os equipamentos da empresa. No entanto, os processos gerenciais se relacionam com a governança corporativa, a formulação da estratégia, o estabelecimento de metas de fornecimento do produto, o planejamento para fornecer o produto, a definição de diretrizes e procedimentos organizacionais e a manutenção das informações e das competências necessárias à tomada de decisão. O terceiro tipo de processo adquire uma posição de destaque para Rummler e Brache (1995) em função da importante atribuição de orientação e coordenação junto aos outros dois tipos de processos.

Gonçalves (2000) define processo como qualquer atividade ou conjunto de atividades que recebe um "input", adiciona valor a ele e fornece um "output" a um cliente específico. Ele também classifica os processos das organizações em três categorias:

- Processos Primários, identificados como aqueles que caracterizam a estratégia de atuação da empresa e geram um produto ou serviço para o cliente externo. Tais processos assumem especial importância, pois eles são ligados à essência do funcionamento da organização e é por meio deles que se pode agregar valor ao cliente. Nessa categoria Gonçalves cita genericamente os processos de desenvolvimento de produto, vendas e distribuição, atendimento de pedidos e atendimento de garantia;

- Processos Organizacionais ou de Suporte, aqueles que garantem o suporte adequado à operação dos processos primários do negócio. Tais processos geralmente produzem resultados imperceptíveis para os clientes externos, mas são essenciais para condução do negócio. Entre as atividades pertinentes a esses processos podem ser incluídos o recrutamento e seleção de empregados, suprimentos e o controle da qualidade do produto;

- Processos Gerenciais, aqueles que são direcionados aos gerentes e suas relações e tomadas de decisões, incluindo as ações de medição e ajuste do desempenho. Tais processos abrangem atividades como o planejamento estratégico, a definição das metas departamentais, alocação de recursos para a execução das atividades, avaliação de resultados e gestão das interfaces com as diversas áreas da organização. 
A norma francesa AFNOR FD X 50-176 (2005) foi estabelecida com o objetivo de incentivar a construção e a melhoria do sistema de gestão por parte da administração, servindo como uma ferramenta de apoio à tomada de decisão e apresenta os fundamentos da abordagem por processo aplicáveis a qualquer tipo de organização, independentemente do seu tipo, porte e setor de atuação. Esta norma aponta que a classificação dos processos do negócio em famílias pode ser útil para facilitar a sua identificação e sugere o seguinte agrupamento:

- Processos de realização, aqueles que contribuem diretamente para a realização do produto, desde a detecção das necessidades dos clientes até a sua satisfação. Eles incluem todas as atividades relacionadas ao ciclo de realização do produto;

- Processos de apoio ou suporte, essenciais para a condução dos processos de realização, fornecendo-lhes todos os recursos necessários. Eles incluem atividades relacionadas ao suprimento de recursos humanos, financeiros, materiais (instalações e manutenção patrimonial, de equipamentos, de hardware, de software, etc.) e ao processamento das informações. Dependendo da finalidade, do tipo de produto e dos clientes, o mesmo tipo de processo considerado como um processo de realização numa organização pode ser considerado como processo de suporte noutra; - Processos de gestão ou gerenciais, que compreendem a definição das políticas, dos objetivos, de diretrizes e de instruções para alocação de recursos na organização. Eles asseguram a coerência e a integração entre os processos de realização e de suporte. Eles incluem a medição e o monitoramento dos resultados e seu uso adequado para obtenção da melhoria do desempenho. - A Fundação Nacional da Qualidade - FNQ (2011) adota a definição de processo como um conjunto de atividades inter-relacionadas ou interativas que transformam insumos (entradas) em produtos (saídas). Conforme a FNQ os processos são classificados em três tipos:

- Processos principais do negócio, aqueles que, com suas operações, agregam valor diretamente para os clientes. Estão envolvidos na geração do produto e na sua venda e transferência para o cliente, bem como na assistência pós-venda e disposição final. Os processos principais do negócio são também conhecidos como processos finalísticos, processos primários ou processos-fim; - Processos de apoio, aqueles que sustentam, com suas operações, os processos principais do negócio e a si mesmos, fornecendo bens e serviços;

- Processos gerenciais ou de gestão, aqueles de natureza gerencial, não operacional, e que são requeridos nos critérios de excelência do Prêmio Nacional da Qualidade. Também conhecidos como práticas de gestão possuem regras de funcionamento denominadas padrões de trabalho que podem ser encontradas na forma de políticas, princípios, normas internas, procedimentos, rotinas, normas administrativas, fluxogramas, comportamentos coletivos ou qualquer meio que permita orientar a execução das atividades dos demais processos.

Portanto, de acordo com a subdivisão dos processos do negócio proposta pelos autores citados anteriormente, os processos de gestão, ou gerenciais, são aqueles que asseguram a coerência e a integração dos processos de execução, ou principais do negócio, e os de apoio. Tem um papel fundamental na coordenação e na orientação para a realização dos outros dois tipos de processos. Dizem respeito aos gerentes, suas relações e tomadas de decisões. Sua estruturação adequada é essencial para que os processos principais e de apoio sejam executados de acordo com as diretrizes estabelecidas pela liderança e para medir, monitorar e melhorar o desempenho da organização. Neste aspecto que se enquadra a melhoria do desempenho ambiental das empresas por meio da implantação das oportunidades de produção mais limpa.

\section{I.2 PROdUÇão MAIS LIMPA NA INDÚSTRIA DO PLÁSTICO}

O conceito de produção mais limpa como aplicação contínua de uma estratégia ambiental preventiva e integrada aos processos, produtos e serviços para aumentar a eficiência global e reduzir os riscos para os seres humanos e ao ambiente foi utilizado pela primeira vez, em 1989, pelo Programa das Nações Unidas para o Meio Ambiente (PNUMA) e nos últimos anos vem se incorporando progressivamente à agenda do mundo empresarial.

O princípio básico da produção mais limpa é a eliminação da poluição e dos desperdícios durante o processo de produção, pois todos os resíduos que a empresa gera representam um custo elevado para ela própria, pois foram adquiridos a preço de mercado de matéria-prima, e para a sociedade como 
um todo, pois consumiram insumos cada vez mais escassos como água e energia. Uma vez gerados, continuam a consumir recursos, seja sob a forma de gastos de tratamento e armazenamento, de multas e penalizações pela falta desses cuidados, ou ainda pelos danos à imagem e à reputação da empresa.

Esse conceito foi desenvolvido, como mais um desafio de melhoria contínua, por empresas líderes na indústria, para se tornar parte integrante do negócio (GIANNETTI E ALMEIDA, 2006). A produção mais limpa pode ser aplicada aos processos utilizados em qualquer indústria, aos seus produtos e aos vários serviços fornecidos na sociedade. É um termo amplo que engloba outros termos associados como eco-eficiência, prevenção da poluição (P2), minimização de resíduos e produtividade verde. Na sua essência a aplicação de produção mais limpa protege o ambiente, o consumidor e o trabalhador, enquanto melhora a eficiência, a lucratividade e a competitividade industrial (UNIDO, 2013).

A produção mais limpa visa a redução dos impactos ambientais, de saúde e de segurança dos produtos ao longo do seu ciclo de vida, desde a extração de matérias-primas, passando pela fabricação e utilização, até a disposição final do produto. Implica na incorporação das preocupações ambientais na concepção e prestação de serviços e resulta na conservação de matérias-primas, água e energia; eliminação de substâncias tóxicas e materiais perigosos; redução da quantidade e toxidade de todas as emissões e resíduos durante o processo de produção.

Por essa razão, a iniciativa da Companhia Ambiental do Estado de São Paulo (CETESB) e do Sindicato da Indústria de Material Plástico do Estado de São Paulo (SINDIPLAST) tem especial relevância pois a indústria de transformação de plásticos é a sétima maior da economia nacional e impacta em praticamente todas as cadeias produtivas. A publicação do Guia Técnico Ambiental da Indústria de Transformação e Reciclagem de Materiais Plásticos (CETESB, 2011) responde ao unânime diagnóstico mundial de que o caminho da prosperidade socioeconômica passa, necessariamente, pelo crescimento sustentado da atividade industrial.

O guia integra uma série de publicações destinadas a contribuir com os preceitos de desenvolvimento sustentável. Em suas páginas são encontrados detalhes sobre a aplicação prática do conceito de produção mais limpa e foi especialmente elaborado por um grupo de especialistas do setor para disseminar informações e dar diretrizes para empresários e colaboradores que atuam direta ou indiretamente com a indústria de transformação do plástico, bem como aos demais interessados em entender os caminhos que podem levar à preservação do meio ambiente.

No entanto, de acordo com o histórico das ações nos diversos setores, esta iniciativa merece uma ação que complemente esse esforço dos técnicos e especialistas dos processos de transformação do plástico e incorpore nos processos gerenciais das empresas do setor os fundamentos da excelência em gestão que permitirão obter melhorias permanentes na eficiência dos processos produtivos, na minimização dos impactos ambientais e nos resultados dos indicadores de desempenho ambiental.

\section{METODOLOGIA}

Para verificação da proposição da pesquisa e consecução dos seus objetivos, as atividades foram subdivididas em duas etapas:

I - Revisão da Literatura e Construção do Modelo;

II - Pesquisa Quantitativa sobre Produção Mais Limpa no Setor de Transformação de Plástico (aplicação do questionário junto aos associados do SINDIPLAST)

$\mathrm{Na}$ construção de um modelo que permita avaliar o relacionamento entre processos gerenciais estruturados (qualidade da gestão ambiental) e os resultados de indicadores ambientais decorrentes das iniciativas implantadas de produção mais limpa foi realizada uma extensa pesquisa bibliográfica sobre processos organizacionais, seus tipos e suas classificações. Também foram considerados os requisitos utilizados nos modelos de excelência em gestão, principalmente os adotados nas premiações nacionais e internacionais mais relevantes, e indicadores de desempenho utilizados na gestão ambiental. Com base nesta revisão foi elaborado o questionário de pesquisa quantitativa para aplicação na etapa II.

A pesquisa quantitativa foi realizada junto aos associados do Sindicato da Indústria de Material Plástico do Estado de São Paulo (SINDIPLAST). Cerca de 50 associados foram convidados para uma palestra sobre produção mais limpa no auditório do sindicato e na oportunidade foi aplicado o ques- 
tionário de pesquisa contendo dez afirmações sobre gestão ambiental, nove sobre processos gerenciais existentes e uma sobre resultados obtidos dos indicadores de desempenho ambiental.

Para analisar as respostas da pesquisa quantitativa foi aplicado o teste de homogeneidade pelo Qui Quadrado em tabelas de contingência (COSTA NETO, 2002), para testar a compatibilidade entre as respostas a cada uma das nove primeiras afirmações, consideradas de aplicação das boas práticas gerenciais, com a décima questão, representativa dos resultados de Produção Mais Limpa. O teste do Qui Quadrado é um teste de hipóteses que se destina a encontrar um valor da dispersão para duas variáveis nominais, avaliando a associação existente entre variáveis qualitativas. É um teste não paramétrico, ou seja, não depende dos parâmetros populacionais, como média e variância. O princípio básico deste método é comparar proporções, isto é, as possíveis divergências entre as frequências observadas e esperadas para certo evento.

Evidentemente, pode-se dizer que dois grupos se comportam de forma semelhante se as diferenças entre as frequências observadas e as esperadas em cada categoria forem muito pequenas, ou seja, próximas a zero. Portanto, o teste é utilizado para verificar se a frequência com que um determinado acontecimento observado em uma amostra se desvia significativamente ou não da frequência com que ele é esperado; e para comparar a distribuição de diversos acontecimentos em diferentes amostras, a fim de avaliar se as proporções observadas destes eventos mostram ou não diferenças significativas ou se as amostras diferem significativamente quanto às proporções desses acontecimentos.

Quanto maior o valor da estatística de teste Qui Quadrado maior a incoerência encontrada. Os valores críticos do Qui Quadrado, com dois graus de liberdade, foram verificados com 1\%, 5\% e 10\% de significância. As afirmações em que a homogeneidade foi aceita com maior conviç̧ão são aquelas que apresentaram dispersões próximas a zero entre as frequências esperadas e as observadas. Espera-se que a maioria das afirmações sobre a existência de processos gerenciais estruturados se relacione positivamente com os resultados ambientais obtidos pela empresa com a aplicação de oportunidades de produção mais limpa para que seja possível confirmar a hipótese.

\section{RESULTADOS E DISCUSSÃO}

\section{I REVISÃo DA LITERATURA}

Levando-se em consideração o resultado da revisão da literatura sobre processos organizacionais e as distintas formas de classificação de cada um dos autores pesquisados, apresenta-se um quadro resumo resultante da compilação das suas ideias, adotando a frequência das aparições em cada tipo de processo como critério para a escolha das atividades a serem consideradas. A tabela 1 mostra a classificação de cada um dos autores pesquisados para os tipos de processos organizacionais e a tabela 2 apresenta o resultado da compilação em três tipos de macroprocessos de uma organização e os processos e atividades que compõem cada um deles.

Com base na revisão da literatura, o modelo de avaliação da qualidade da gestão ambiental foi desenvolvido para o projeto de pesquisa, considerando-se também os modelos de excelência existentes. Os modelos utilizados pelo Malcolm Baldrige National Quality Award (BNQP, 2011/2012), The European Quality Award (EFQM, 2010), Prêmio Nacional da Qualidade (FNQ, 2011) e demais premiações alinhadas aos critérios de excelência em gestão foram desenvolvidos no final da década de 80 para identificar organizações de gestão exemplar, ou seja, práticas de gestão e padrões de trabalho estruturados, gerando bons resultados em todas as perspectivas importantes para o negócio. Além dos prêmios servirem como reconhecimentos às organizações que possuem práticas exemplares e bons resultados, o grande benefício aliado aos modelos é a possibilidade de analisar as organizações sob a ótica da qualidade da gestão, isto é, de seus processos gerenciais.

Tendo por base os processos e as atividades que compõem os macroprocessos gerenciais, apresentados na Tabela 2, e considerando-se os modelos de excelência em gestão adotados pelos prêmios de qualidade na gestão, foi desenhado o questionário da pesquisa qualitativa (Afirmações da Tabela 3)para aplicação na etapa II.

\subsection{Pesquisa QUalitativa}

O SINDIPLAST reuniu cerca de 50 empresas do setor de transformação de plásticos para apresentar conceitos básicos sobre produção mais limpa e excelência em gestão. No final da apresen- 
Tabela 1 - Classificação dos tipos de processos organizacionais

\begin{tabular}{|c|c|c|c|}
\hline \multirow{2}{*}{ Auter } & \multicolumn{3}{|c|}{ CLASSIFICACXO DOS PROCESSOS } \\
\hline & PRINCIPAIS & APOIO & GERENCIAIS \\
\hline 尊学 & 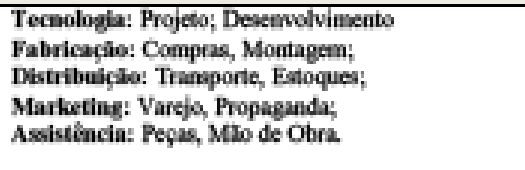 & - & - \\
\hline$\frac{8}{8}$ & 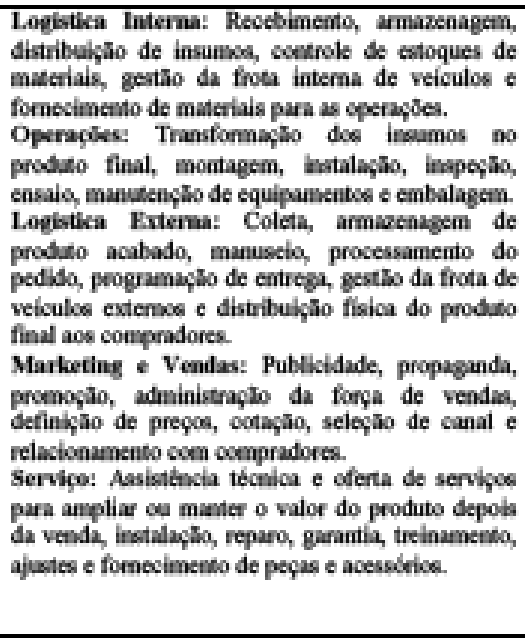 & 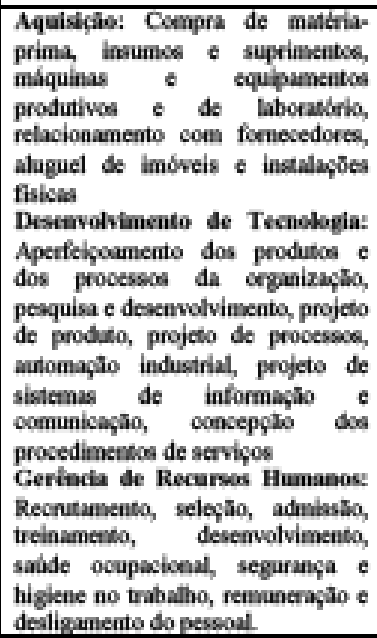 & 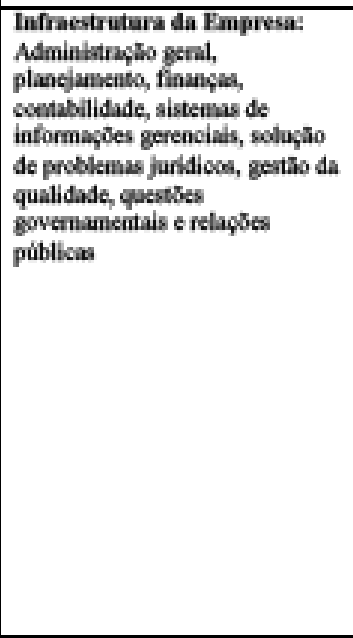 \\
\hline 喥 & 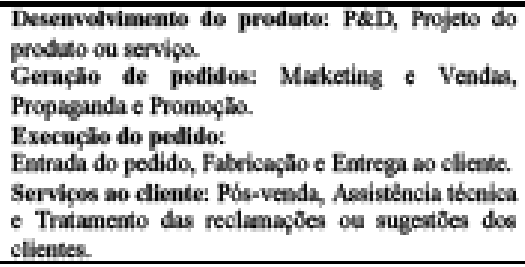 & $\begin{array}{l}\text { Financeire } \\
\text { Recurses Humanes } \\
\text { Compras }\end{array}$ & - \\
\hline 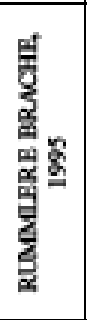 & $\begin{array}{l}\text { Vender, } \\
\text { Produzir, } \\
\text { Entregar, } \\
\text { Dar asaistencia ténica, } \\
\text { Aperfeiçoar } \\
\text { Descontinuar o produto }\end{array}$ & $\begin{array}{l}\text { Comprar materiais, } \\
\text { Coentrolar os recursos para a } \\
\text { execuçlo do produlo, } \\
\text { Recrutar e selecionar peseoal, } \\
\text { Fazer a contabilidade, } \\
\text { Manter of equipaenentios da } \\
\text { empresa. }\end{array}$ & 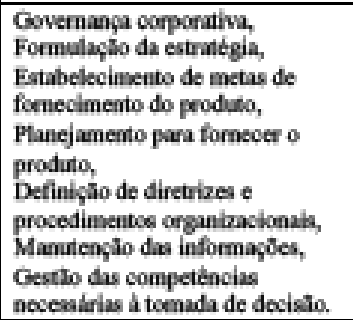 \\
\hline 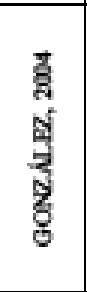 & 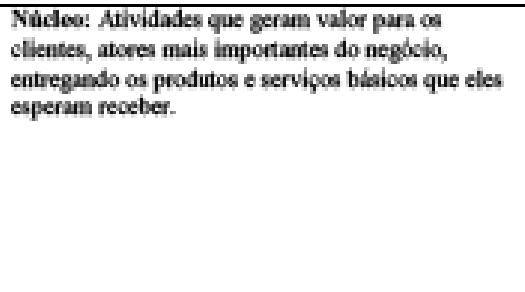 & 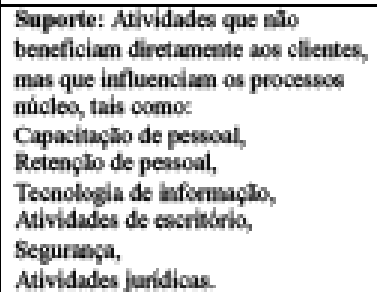 & 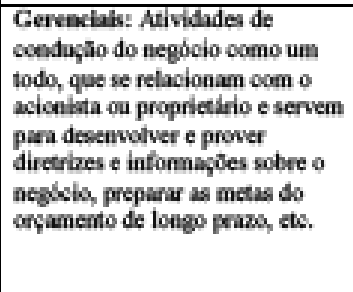 \\
\hline 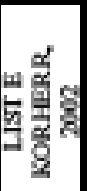 & 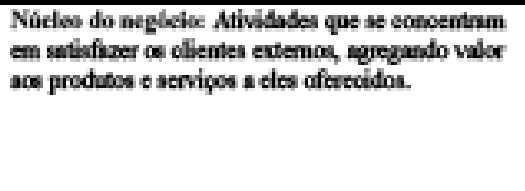 & 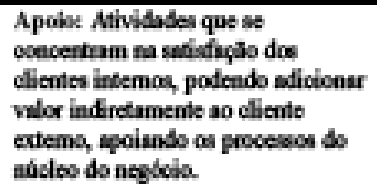 & $\begin{array}{l}\text { Gestlice Atividades que se } \\
\text { peeosupam cem o gerenciamento } \\
\text { don peosesacs do absleo cu de } \\
\text { apolo, cu com o planejumento do } \\
\text { segócio so nivel emprearial. }\end{array}$ \\
\hline $\begin{array}{l}\frac{8}{7} \\
\text { d. } \\
\frac{3}{2} \\
\frac{3}{8}\end{array}$ &  & 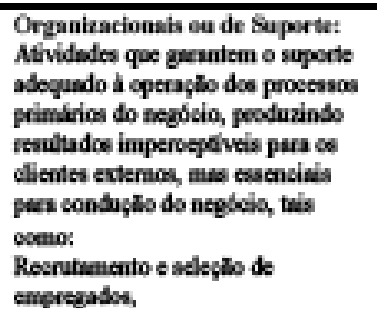 & 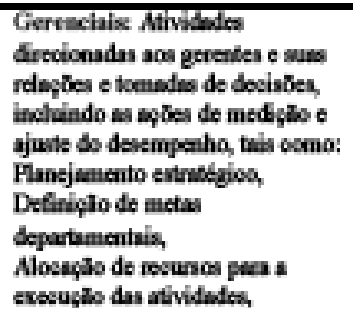 \\
\hline
\end{tabular}




\begin{tabular}{|c|c|c|c|}
\hline 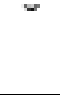 & & $\begin{array}{l}\text { Treinumento do peasoul, } \\
\text { Suprimentos, } \\
\text { Aviliagiso da qualidade do peoduta. }\end{array}$ & 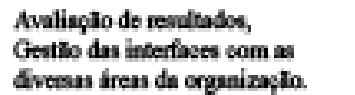 \\
\hline 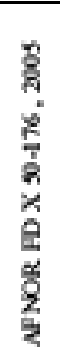 & 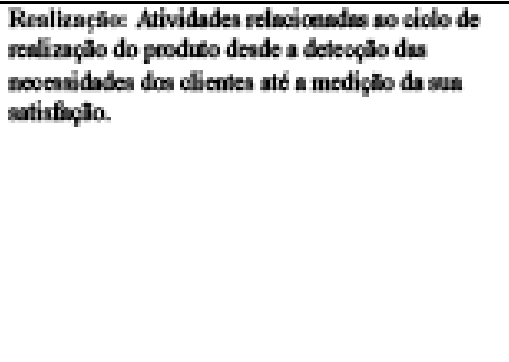 & 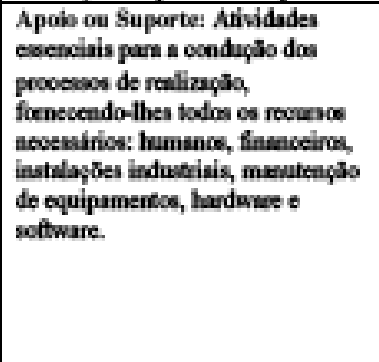 & 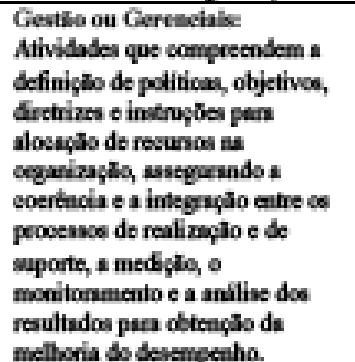 \\
\hline 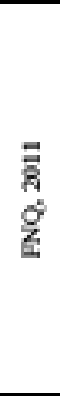 & 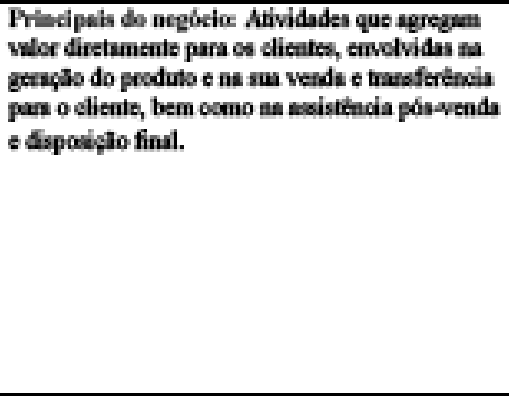 & 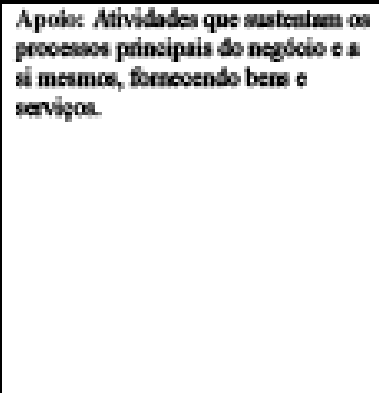 & 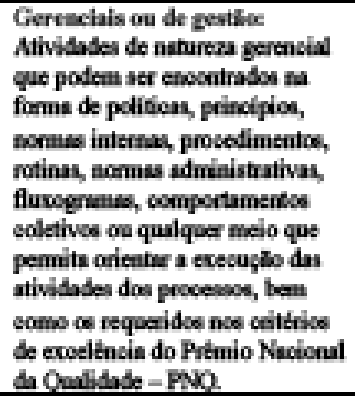 \\
\hline
\end{tabular}

Fonte: Revisão da literatura pelos autores

Tabela 2 - Os três tipos de macroprocessos de uma organização

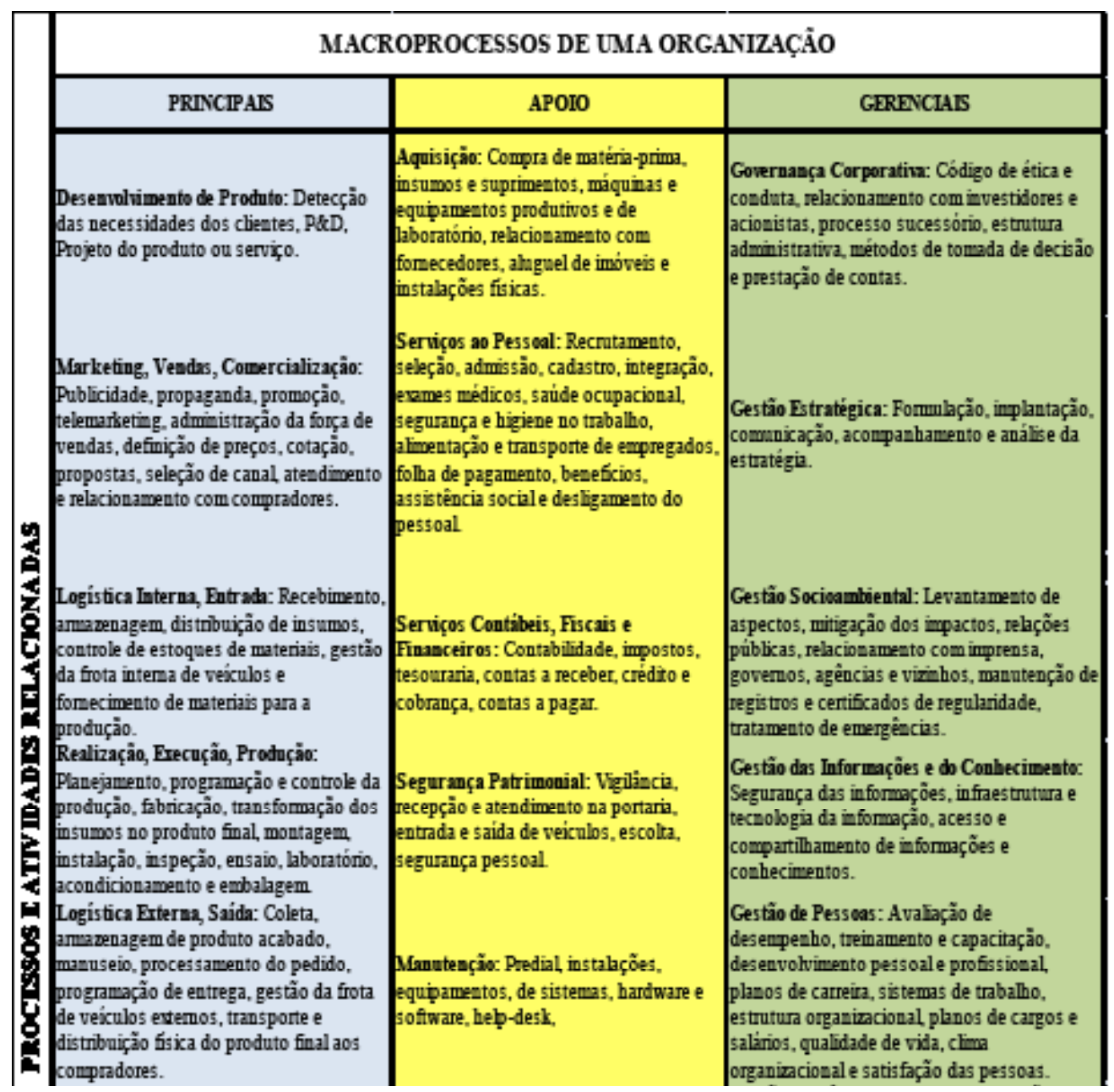




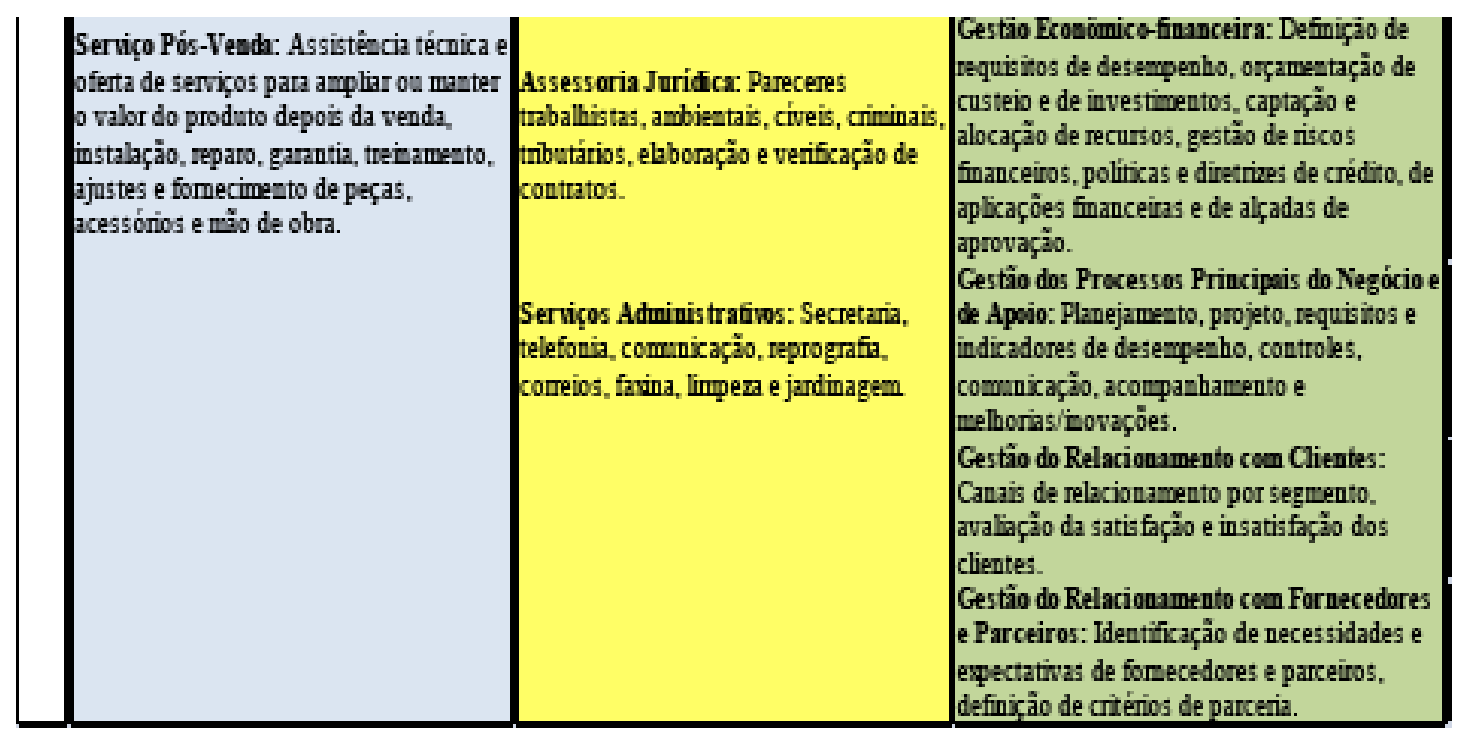

Fonte: Elaborada pelos autores

tação, o questionário foi explicado e distribuído a todos os participantes da seção de treinamento e 32 executivos representantes de empresas associadas ao sindicato concordaram em responder. A pesquisa continha dez afirmações que resumiam o modelo de avaliação desenvolvido (nove sobre os processos gerenciais da empresa e uma sobre resultados ambientais), como mostra a tabela 3.

Tabela 3 - As 10 afirmações da pesquisa e o número de respostas dos participantes

\begin{tabular}{|c|c|c|c|c|}
\hline \multirow[b]{2}{*}{ AFIRMAÇŌES } & \multicolumn{4}{|c|}{ NoDE RESPOSTAS } \\
\hline & $\overline{\mathbf{D}}$ & DP & $C P$ & $\bar{c}$ \\
\hline 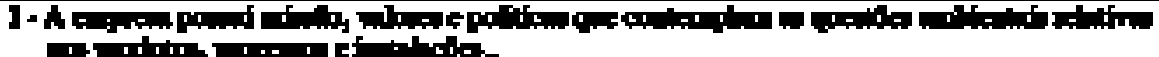 & $\mathbf{d}$ & 6 & $\mathbf{1}$ & 16 \\
\hline in & 4 & 9 & $\mathbf{4}$ & j \\
\hline 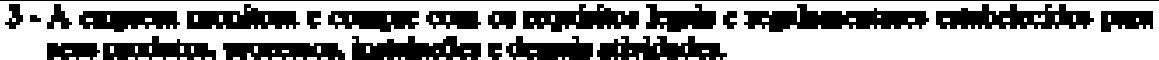 & $\mathbf{I}$ & 2 & $\mathbf{4}$ & W \\
\hline 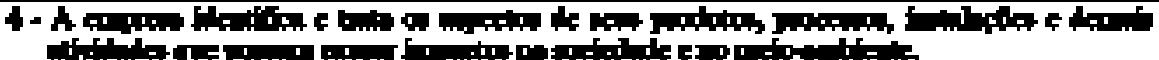 & d & $\mathbf{J}$ & L6 & I0 \\
\hline 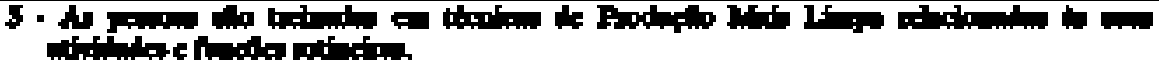 & I & 5 & 16 & I0 \\
\hline D象 & 3 & J] & $\bullet$ & 4 \\
\hline & 4 & 8 & 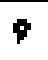 & II \\
\hline & $\mathbf{I}$ & 5 & W & La \\
\hline on & 4 & 7 & $\mathbf{1}$ & 5 \\
\hline 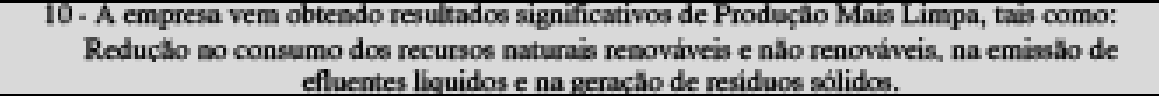 & 3 & 9 & 8 & 12 \\
\hline
\end{tabular}

Os participantes foram solicitados a escolher apenas a resposta que corresponde a alternativa mais adequada para a situação atual de suas empresas. As alternativas foram apresentados em uma escala Likert de 4 pontos, ou seja:

$\mathrm{D}=$ discordo totalmente $\mathrm{DP}=$ Discordo parcialmente, $\mathrm{CP}=$ Concordo Parcialmente, $\mathrm{C}=$ Concordo plenamente.

A tabela 3 mostra o número total de respostas compiladas a partir dos 32 questionários 
preenchidos. Como citado anteriormente, para analisar as respostas foi utilizado o teste de homogeneidade do qui quadrado $(\chi 2)$ por meio de tabelas de contingência, conforme descrito em Costa Neto (2002). O princípio básico deste método é comparar proporções, isto é, as possíveis divergências entre as frequências observadas e esperadas para um determinado evento. Os resultados das respostas D e DP foram agrupados para satisfazer a condição de teste, ou seja, as frequências esperadas sejam maiores ou iguais a 5. Cada uma das nove tabelas de contingência utilizadas para este fim tem a configuração apresentada na Tabela 4.

A variável de teste é dada pela equação 1:

$$
x_{\mathbf{z}}^{\mathbf{z}}=\mathbf{Z} \mathbf{Z} \frac{\left(\mathbf{0}_{i j}-\boldsymbol{E}_{i j}\right)^{\mathbf{z}}}{\boldsymbol{F}_{i j}}
$$

onde $\mathrm{O}_{\mathrm{ij}}$ são as frequências observadas na pesquisa e $\mathrm{E}_{\mathrm{ij}}$ são as frequências teóricas esperadas sob a hipótese de homogeneidade.

A hipótese testada em cada caso é da existência de coerência das respostas quanto às aplicações e os resultados alcançados.

Tabela 4 - Tabela de contingência genérica, i $=1,2, \ldots, 9$

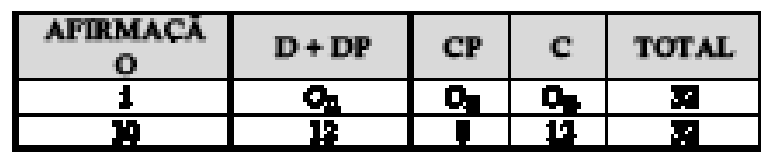

A tabela 5 apresenta os graus de significâncias obtidos nos testes. Quanto maior o valor da estatística de teste $\chi^{2}$ maior a incoerência encontrada. A coluna $\mathrm{R}$ representa o "ranking" decrescente de significância dos resultados. Sendo os valores críticos do $\chi^{2}$ com dois graus de liberdade válidos para esse teste com $1 \%, 5 \%$ e $10 \%$ de significância de, respectivamente $9,210,5,991$ e 4,605, a afirmação 4 teve discordância significativa ao nível $1 \%$, a afirmação 3 ao nível de $5 \%$ e as afirmações 5 e 6 , ao nível de $10 \%$ de significância. As afirmações 1, 2, 7, 8 e 9 foram aquelas em que a homogeneidade foi aceita com maior convicção. É interessante notar que as afirmações 3 e 4 , relacionadas com aspectos legais e de impactos socioambientais, foram aquelas que tiveram mais discordância com os resultados. Isto pode indicar que esses aspectos gerenciais envolvendo temas ligados ao cumprimento da legislação e ao tratamento de aspectos que causam impacto no meio ambiente e na sociedade são tratados por quase todos, independentemente da busca de resultados. Por outro lado, verificou-se coerência entre os resultados e os aspectos ambientais apontados na missão, nos valores e na visão de futuro das empresas, o uso de ferramentas e métodos de produção adequados para tanto e a destinação de recursos financeiros necessários à produção mais limpa.

Tabela 5 - Significância das dependências entre aplicações e resultados

\begin{tabular}{|c|c|c|c|c|c|c|}
\hline AFIRMACCOES & D + DP & $\mathrm{CP}$ & $\mathbf{C}$ & TOTAL & $x_{5}^{2}$ & $\mathbf{R}$ \\
\hline ] & a & $\overline{\mathbf{8}}$ & $\overline{16}$ & $\overline{\mathbf{3}}$ & 1.572 & $\overline{-}$ \\
\hline 2 & 13 & 14 & 5 & 3․ & 450 & - \\
\hline 3 & 3 & 14 & 15 & 포 & 3.3 .9 & 2 \\
\hline 4 & 3 & 5 & 14 & $\overline{\mathbf{3}}$ & 10.06 & $\mathbf{1}$ \\
\hline 5 & $\mathbf{g}$ & 16 & 10 & 포 & 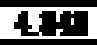 & 4 \\
\hline 6 & {$[9$} & g & 7 & Si & $5 x:$ & 3 \\
\hline 7 & $\mathbf{1 2}$ & 9 & 11 & 32 & 0.101 & $\begin{array}{lll}- & & \\
\end{array}$ \\
\hline 8 & $\mathbf{d}$ & BS & T] & 这 & 3254 & - \\
\hline g & $\mathbf{1 1}$ & 12 & 9 & 32 & $12 \pi 2$ & - \\
\hline 10 & $\overline{\mathbf{2}}$ & 8 & $\mathbf{2}$ & 포 & $=$ & - \\
\hline
\end{tabular}




\section{CONCLUSÕES}

O estudo foi concebido para desenvolver um modelo de avaliação da qualidade da gestão ambiental das empresas da indústria do plástico e para comprovar a proposição de que os resultados ambientais permanentes de produção mais limpa tem uma relação positiva com a existência de processos gerenciais estruturados e padronizados nas empresas. A relação positiva entre o desempenho ambiental e a existência de processos gerenciais estruturados foi encontrada utilizando-se o modelo de avaliação desenvolvido e aplicado numa pesquisa quantitativa realizada com 32 empresas associadas ao SINDIPLAST.

Ficou claro que a iniciativa da elaboração de guias técnicos de produção mais limpa para a indústria foi um passo importante para a comunicação técnica e conscientização dos colaboradores. No entanto, apenas as empresas que integraram os conceitos e princípios de produção mais limpa em seus processos gerenciais e, consequentemente, nos processos principais do negócio e de apoio, alcançaram resultados positivos e permanentes nos indicadores ambientais.

A gestão estruturada e padronizada, com sua abrangência holística, possibilita o refinamento dos processos por meio do aprendizado organizacional, além de tornar claro o papel dos gestores nas organizações. Este é o grande diferencial das empresas do setor do plástico de São Paulo que atingem resultados em relação às que não o fazem. É importante salientar que este estudo precisa se estender a uma maior quantidade de empresas e novos setores da indústria para possibilitar a discussão sobre a generalização dos resultados.

\section{AGRADECIMENTOS}

Os autores agradecem o apoio financeiro na forma de bolsa de doutorado para o PROSUP da CAPES. Agradecimentos especiais são dirigidos ao SINDIPLAST pela cooperação na obtenção de dados e informações para a realização deste estudo.

\section{REFERÊNCIAS}

ADAIR, C.; MURRAY, B. Revolução total dos processos. São Paulo: Nobel, 1996.

AFNOR - Association Française de Normalisation. FD X 50-176. Management des processus. La Plaine St Denis Cedex, 2005.

ASHTON, W.; LUQUE, A.; EHRENFELD, J. R. Best practices in cleaner production promotion and implementation for smaller enterprises. Prepared for Multilateral Investment Fund (MIF) and Interamerican Development Bank (IADB), Washington, DC. School of Forestry and Environmental Studies, Yale University, New Haven, CT - USA, 2002.

BNQP - BALDRIGE NATIONAL QUALITY PROGRAM. Criteria for performance excellence. Gaithersburg, MD: Baldrige National Quality Program, 2011-2012.

BONILLA, S. H.; ALMEIDA, C. M. V. B.; GIANNETTI, B. F.; HUISINGH, D. The roles of cleaner production in the sustainable development of modern societies: an introduction to this special issue. Journal of Cleaner Production, vol. 18, page 1-5, 2010.

CETESB. Guia Ambiental da Indústria de Transformação e Reciclagem de Materiais Plásticos. Série P+L, São Paulo, 2011.

COSTA NETO, P. L. O. Estatística. Edgard Blucher, São Paulo, 2ª ed., 2002.

EHRLICH, P. R.; EHRLICH, A. H. The Culture Gap and Its Needed Closures. International Journal of Environmental Studies, vol. 67, n. 4, 2010. 
EFQM - EUROPEAN FOUNDATION FOR QUALITY MANAGEMENT. O modelo de excelência da EFQM: versão grandes empresas, unidades operacionais e de negócio. Brussels: European Foundation for Quality Management, 2010.

FNQ - Fundação Nacional da Qualidade. Critérios de excelência: avaliação e diagnóstico da gestão organizacional. 19a Edição, São Paulo, 2011.

GIANNETTI, B. F.; ALMEIDA, C. M. V. B. Ecologia industrial: conceitos, ferramentas e aplicações. Edgard Blucher, São Paulo. 2006.

GONÇALVES, J. E. L. As empresas são grandes coleções de processos. Revista de Administração de Empresas - RAE, São Paulo, v. 40, p. 6-19. Jan/Mar 2000.

GONZÁLEZ, A. H. Identificación de procesos de negocio. Revista Ingeniería Industrial, La Habana, vol. $\mathrm{XXV}, \mathrm{n}^{\circ} 3,2004$.

HART, S. L. Beyond Greening: Strategies for a Sustainable World. Harvard Business Review. page 66-76, January-February, 1997.

LIST, B.; KORHERR, B. A UML - Unified Modeling Language - 2 Profile for Business Process Modelling. Research funded by the Austrian Federal Ministry for Education, Science, and Culture, and the European Social Fund (ESF) under grant 31.963/46-VII/9/2002.

MCKINSEY \& COMPANY. Competitive cost analysis. Research Report by Carter F. Bales, P. C. Chatterjee, Donald Gogel and Anupam Puri, March 1980.

PORTER, M. E. Competitive advantage: creating and sustaining superior performance. New York: Free Press, 1985.

RUMMLER, G. A.; BRACHE, A. P. Improving Performance: How to manage the white space on the organizational chart. San Francisco: Jossey-Bass, 1995.

UNIDO - United Nations Industrial Development Organization. Cleaner Production. Disponível em: http:// www.unido.org/index.php?id=o5152. Acessado em: 12/01/2013.

VENDRAMETTO, O.; PALMERI, N.; OLIVEIRA NETO, G. C.; PERRETI, O. D. Cleaner Production: A Growing Movement In Brazilian Companies. Produção Online, vol. 10, n. 1, page 49-70, 2010. 